\title{
L'hémisphère cérébral droit : un atout en anglais de spécialité
}

Isabelle Hesling

\section{OpenEdition}

\section{Journals}

Édition électronique

URL : http://journals.openedition.org/asp/1541

DOI : 10.4000/asp. 1541

ISBN : 978-2-8218-0390-9

ISSN : 2108-6354

Éditeur

Groupe d'étude et de recherche en anglais de spécialité

Édition imprimée

Date de publication : 30 décembre 2002

Pagination : 121-140

ISSN : 1246-8185

Référence électronique

Isabelle Hesling, "L'hémisphère cérébral droit : un atout en anglais de spécialité », ASp [En ligne], 37-38 | 2002, mis en ligne le 26 juillet 2010, consulté le 30 avril 2019. URL : http://

journals.openedition.org/asp/1541; DOI : 10.4000/asp.1541

Ce document a été généré automatiquement le 30 avril 2019.

Tous droits réservés 


\title{
L'hémisphère cérébral droit : un atout en anglais de spécialité
}

\author{
Isabelle Hesling
}

\section{Recherche fondamentale}

\subsection{Linguistique de la parole}

1 La parole désigne la réalité humaine telle qu'elle se fait jour dans l'expression par le biais de l'expressivité. Cette notion de parole regroupe d'une part les règles syntaxiques, la grammaire et le lexique qui constituent l'expression et d'autre part les intonations, les rythmes et la gestuelle qui constituent l'expressivité (Figure 1). C'est la combinaison de l'expression et de l'expressivité qui permet l'existence de la véritable communication. Les modalités de la communication sont représentées par la somme des modalités verbales et non-verbales, ces deux composantes étant tout aussi importantes l'une que l'autre dans la communication langagière. 


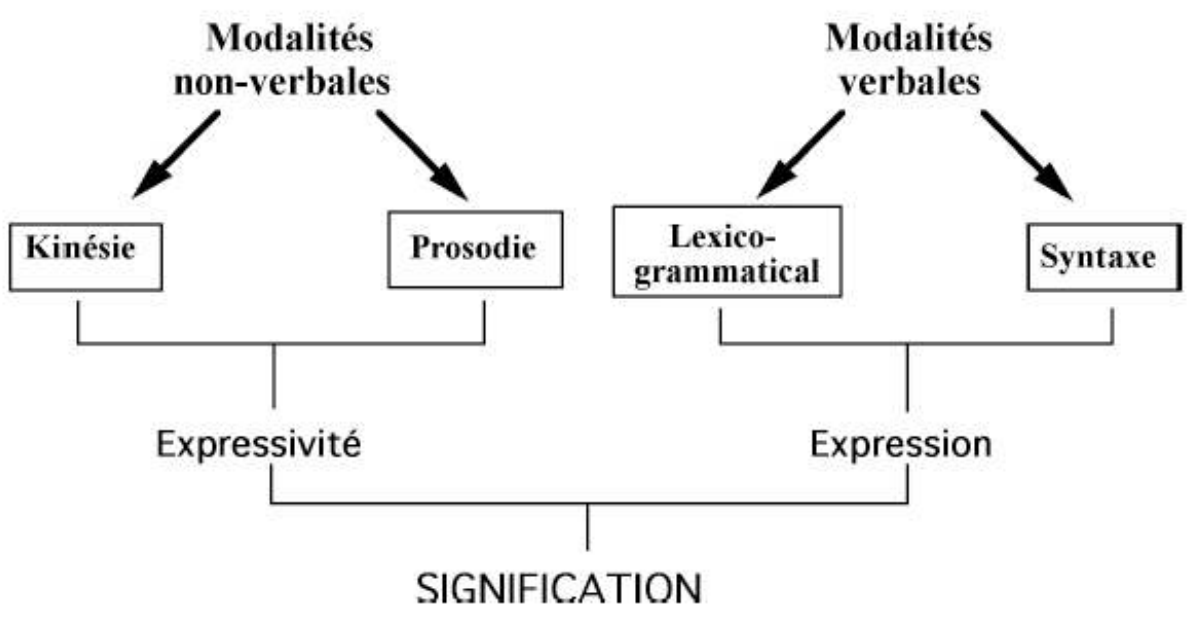

2 Il apparait très clairement que beaucoup de courants linguistiques traitent de l'expression sans faire aucunement référence à l'expressivité. En d'autres termes, ces études portent sur la langue et non pas sur la parole. Bon nombre de linguistes considèrent la forme écrite « statique » de la langue, en occultant le fait que, derrière tout acte de communication, il existe préalablement un sujet pensant et parlant. En faisant abstraction de ce sujet, ces recherches font de ce fait abstraction des modalités nonverbales. La théorie de l'information, le structuralisme saussurien, les linguistiques structuralistes de 1950 à 1970, les courants distributionnalistes et chomskyen, la statistique ont largement contribué à mieux faire connaître l'ossature structurale extérieure des langues. Cependant, l'organisation formelle de la langue dans de tels systèmes est plus importante que la fonction significative et expressive du langage. Dans ces concepts, toute analyse est basée sur la continuité horizontale des mots dans une phrase et représente en somme une structure à une dimension.

3 Selon Gubérina (1984), l'étude de la parole doit être conçue sur la base des rapports réciproques et permanents entre société et individu et doit englober à la fois la cohérence des mots (modalités verbales) et les valeurs de la langue parlée (modalités non-verbales). Une telle structure apparait comme étant pluridimensionnelle : elle est en même temps horizontale (les mots) et verticale car les mots ont pour support le rythme, l'intonation, etc., c'est-à-dire toutes les composantes des valeurs de la langue parlée. « La parole n'est pas une addition pure et simple de plusieurs composantes limitées en nombre, mais une qualité dynamique qui ne rend notre expression ni horizontale, ni linéaire, mais verticale "(Gubérina, in Robergé 1984). "Toute étude de notre expression devrait inclure l'ensemble des moyens dont nous nous servons en parlant » (Gubérina 1984) (Figure 2). 
Figure 2. La Parole (Hesling 2000, adaptée de Gubérina in Robergé 1992)

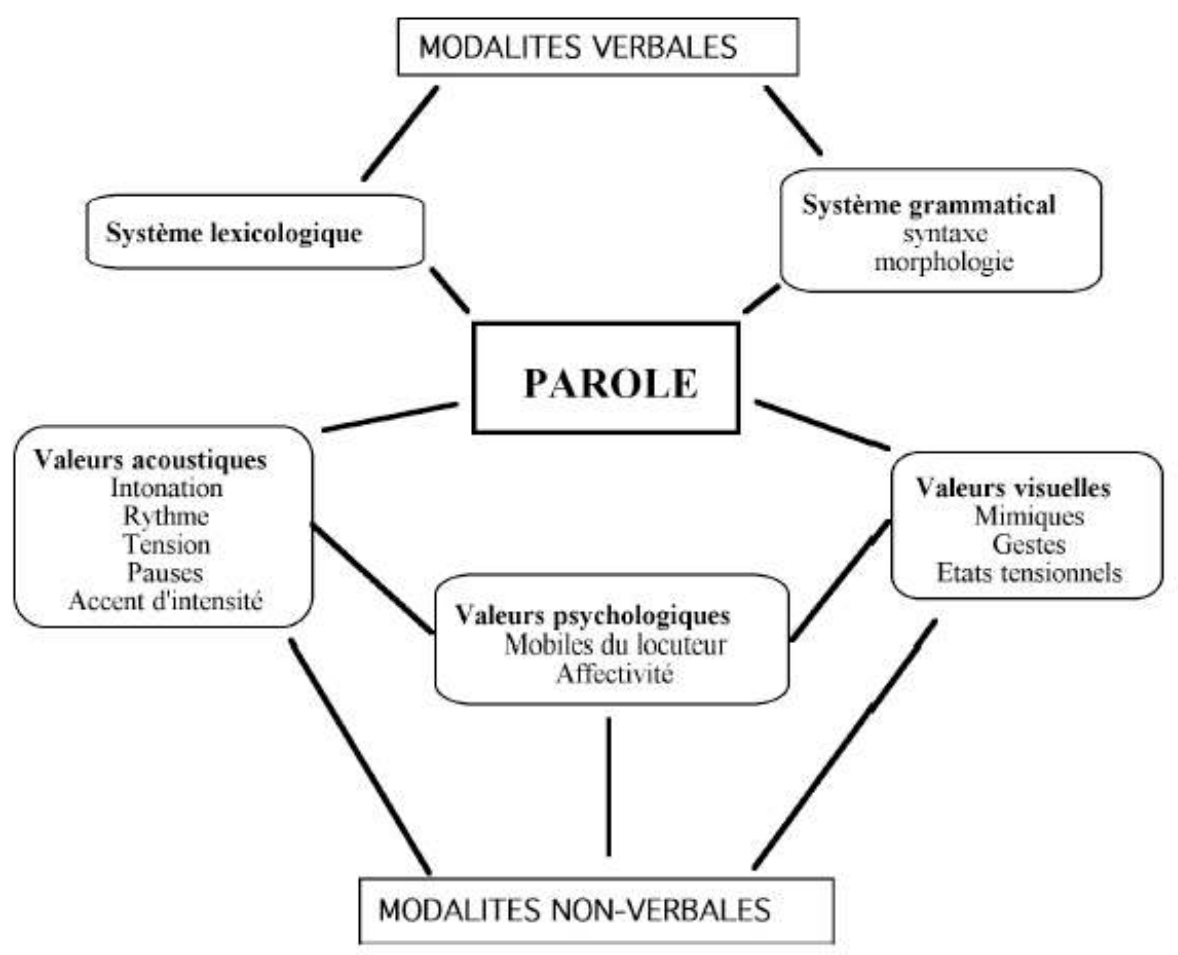

Toutes les caractéristiques de la parole ne s'additionnent pas les unes aux autres comme à l'écrit, car ces composantes de la parole ont un caractère de non-linéarité qui est la condition sine qua non de l'existence de la parole dans le temps et dans l'espace. La parole est perçue et produite de façon globale: la signification intellectuelle (contenu de l'expression) et la signification affective (contenu de l'expressivité) sont perçues en même temps. Cette structure tridimensionnelle se forme donc en fonction de la communication, un équilibre dynamique (et non-statique puisqu'il existe une mouvance permanente) s'établit entre l'individuel et le social. Tous les éléments de communication (éléments structurants) ne sont pas tous toujours présents au même niveau. La structure mobile s'établit en "fonctionnant ", c'est-à-dire que le sujet parlant choisit les éléments qui lui paraissent optimaux pour donner forme à la structure linguistique. C'est donc de l'état affectif du sujet parlant que dépend la réalisation linguistique communicationnelle.

Ainsi que le montrent les figures 1 et 2 , la parole comprend l'ensemble des procédés qui interviennent dans l'utilisation du langage, à savoir: (i) les modalités verbales: le système lexicologique et grammatical (phonétique, morphologique et syntaxique) ; (ii) les modalités non-verbales qui peuvent être divisées en trois catégories: les valeurs acoustiques : rythme, intonation, intensité, tension, pause, tempo de l'énoncé ; les valeurs visuelles : le rôle du corps, position du corps et états tensionnels, et plus particulièrement la position envers autrui et la tension corporelle des co-énonciateurs, mimiques, gestes ; les valeurs psychologiques: les aspects psychologiques de la parole, les mobiles de l'énonciateur où l'affectivité intervient dans l'élaboration de la forme d'expression, la situation, le contexte, l'ambiance sociale, constamment présents dans toute communication langagière. 
Ces valeurs apparaissent le plus clairement dans la langue parlée et font référence à des procédés qui, en situation de communication, ne sont pas constitués par les mots proprement dits. Les mots exprimés sont toujours pénétrés par diverses intonations, accompagnées de gestes, qui les rendent ainsi vivants, compréhensibles et expressifs. Que ce soit dans l'utilisation du mot ou des phrases, les modalités non verbales contribuent directement à la valeur sémantique et syntaxique de l'expression langagière. Le matériel lexicologique n'est qu'une partie de l'expression, partie qui, par ailleurs, traduit le moins les sentiments qui accompagnent les idées exprimées : « $90 \%$ au moins des messages affectifs sont non verbaux » (Goleman 1997). Ce n'est pas le sens lexicologique du mot qui détermine son sens, mais « l'intégration de ce mot parmi les valeurs de la langue parlée » (Gubérina 1984).

\subsection{Acoustique}

7 La production de la parole se traduit par des événements acoustiques déclenchés par des activités physiologiques spécifiques. L'énergie nécessaire à la production de la parole est fournie par le courant d'air expulsé par les poumons sous l'effet du muscle intercostal externe et des muscles abdominaux. L'air non sonorisé ainsi projeté met en mouvement les cordes vocales, situées dans le larynx, qui s'ouvrent et se ferment rapidement. Le courant d'air interrompu par le rapprochement des cordes vocales, puis libéré par leur éloignement, se transforme en une succession de bouffées chargées d'énergie acoustique sonorisée. Ces vibrations des cordes vocales, activité physiologique, se traduisent par la production d'une énergie acoustique sonore correspondant à la fréquence fondamentale, notée Fo. Cette fréquence de base est en fait le nombre d'ouvertures et de fermetures des cordes vocales produit en une seconde. Ensuite, la qualité du son produit évolue en fonction des modifications de la configuration du pharynx, la voix devenant parole au niveau de la cavité buccale grâce à l'action finale du voile du palais, de la langue et des lèvres, organes participant aux changements de formes du circuit (Figure 3).

Figure 3. La parole : activité physiologique et acoustique (Hesling 2000)

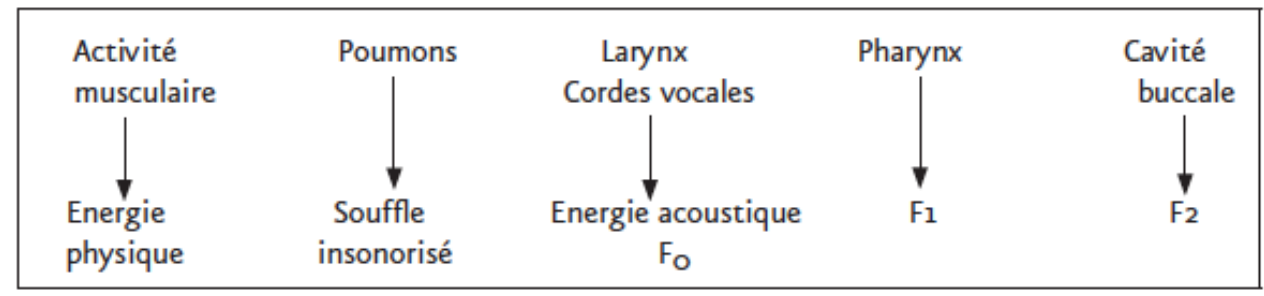

En acoustique, les sons se définissent comme étant des modifications de pression qui se propagent dans l'air, sous la forme d'un mouvement vibratoire, à la vitesse de 340 millisecondes (ms). Les sons purs sont dits simples car ils ne comportent qu'une seule fréquence de vibration. À l'exception de ceux produits par le diapason ou le générateur de fréquences, tous les sons peuvent être définis comme complexes: ils proviennent de l'émission simultanée de multiples fréquences. La parole se distingue des autres sons par des caractéristiques acoustiques qui ont leur origine dans les mécanismes de production (Renard 1983). 
9 Décrire le processus de production de parole en vue de spécifier le résultat acoustique ainsi produit nécessite l'acquisition d'un certain nombre de connaissances liées à la complexité du processus de génération et à ses difficultés de mesure (Calliope 1989) :

1. le signal de parole est essentiellement évolutif: l'analyse centiseconde est généralement reconnue comme constituant un ordre de grandeur raisonnable ;

2. de nombreuses différences anatomiques existent entre les locuteurs et les stratégies articulatoires présentent de grandes variations intra et inter-locuteurs ;

3. le conduit vocal est un "tuyau " tridimensionnel de volume variable difficilement accessible;

4. la source vocale qui excite ce tube acoustique que représente le larynx, est très difficile d'accès et sa fréquence peut évoluer très rapidement (dans une gamme d'une octave autour de $110 \mathrm{~Hz}$ environ pour les hommes et de $210 \mathrm{~Hz}$ pour les femmes) ;

5. le processus de production peut se résumer à une source qui excite un ensemble de cavités ; or l'on ne dispose que du signal produit et non du signal d'excitation (très difficile à évaluer directement); le système étudié (paramètres de source + fonction de transfert du conduit vocal) présente de ce fait plus d'inconnues que de données.

Les progrès de ces dernières années en informatique, ont permis l'élaboration de logiciels d'analyse de parole plus sophistiqués rendant plus précises la distinction et les mesures des différents aspects d'un son. Ainsi, les analyseurs électroniques présentent une représentation tridimensionnelle (temps, fréquence, amplitude) des sons de parole. Le temps est noté en abscisse, les fréquences en ordonnée et l'amplitude, de manière qualitative seulement, par le degré de noirceur du tracé.

11 La partie 1 (Figure 4) représente l'évolution du contour mélodique en Hz. Les phonèmes voisés sont produits par la mise en vibrations des cordes vocales (celles-ci se fermant et s'ouvrant à une certaine vitesse). Tout phonème voisé présente donc des variations de fréquences en fonction de sa durée : ce sont ces variations de F0 qui apparaissent dans cette première partie et qui représentent la courbe mélodique spécifique à chaque énoncé et à chaque locuteur. Les cordes vocales ne vibrant pas lors de la production des sons nonvoisés, les variations de fréquences n'existent pas. La hauteur d'un son dépend de sa fréquence fondamentale (F0). Si la F0 est basse, le son sera perçu grave, si la F0 est élevée, le son sera perçu aigu. 
Figure 4. Les quatre différentes parties du sonagramme de [zip] (Hesling 2000)

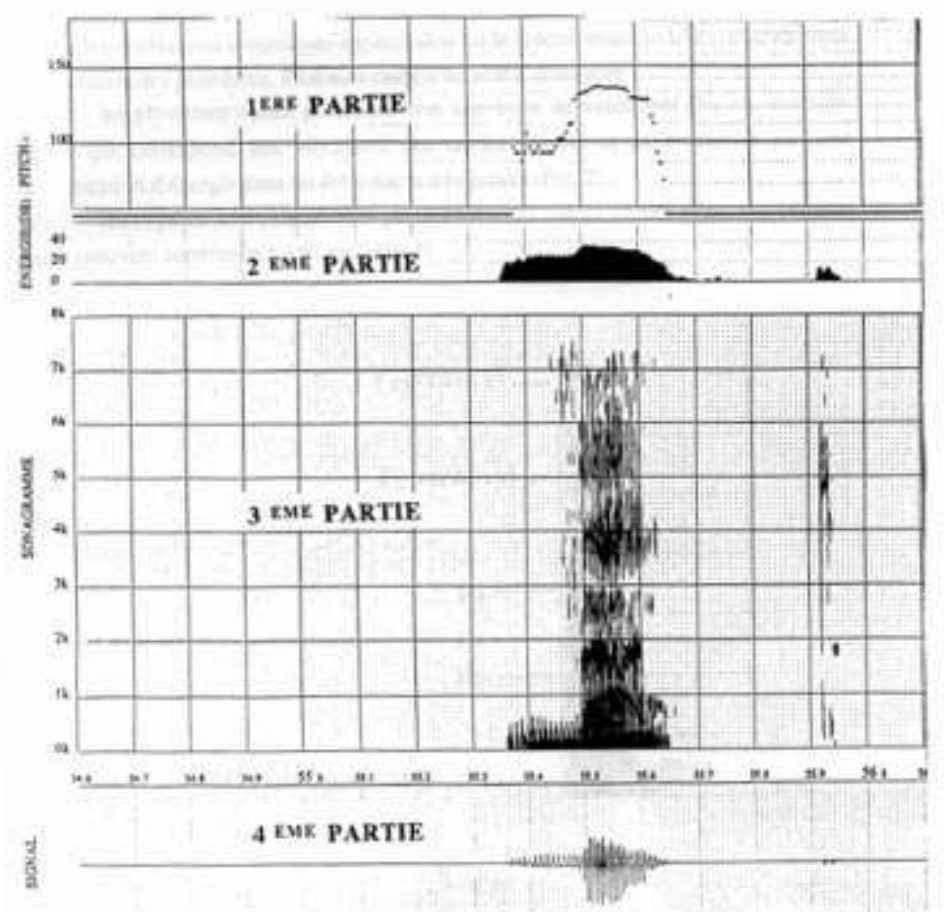

La partie 2 représente l'énergie (ou intensité) mesurée en décibel (Db). D'un point de vue acoustique, l'intensité d'un son résulte de l'amplitude du mouvement vibratoire. L'intensité de la voix est essentiellement due à l'amplitude des vibrations des cordes vocales et à la pression subglottique exercée par l'air pulmonaire.

La partie 3 représente le spectre acoustique de 0 à $8 \mathrm{kHz}$. Le timbre des sons de parole dépend des cavités supraglottiques faisant office de résonateurs. En fonction de la plus ou moins grande densité des fréquences basses par rapport aux fréquences hautes, l'on distinguera le timbre sombre (dominantes basses [u], [v]) du timbre clair (dominantes hautes [i], [s]). Le timbre étant perçu globalement représente forcément une perception subjective.

La partie 4 représente le signal ou l'oscillogramme (forme de l'onde). Certains sons complexes présentent une périodicité (sons voisés). D'autres sons complexes sont apériodiques (sons nonvoisés). S'ils possèdent une certaine durée, ils seront appelés bruits (consonnes fricatives et affriquées non-voisées). S'ils sont très brefs, ils seront appelés impulsions (consonnes occlusives non-voisées). Les sons apériodiques ne possèdent pas de hauteur, puisque la hauteur est liée à la fréquence. 


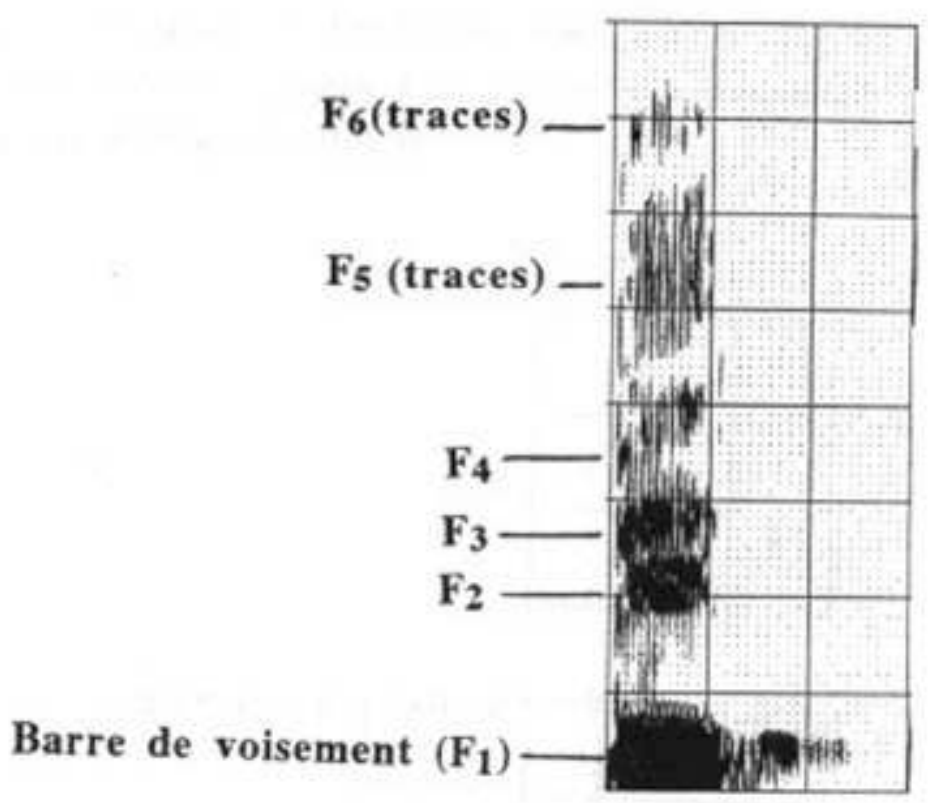

Toutes les réalisations acoustiques apparaissant sur le spectre trouvent leurs origines dans l'articulation des phonèmes. Plusieurs catégories sont à distinguer. Les phonèmes voisés présentent tous une barre de voisement (F1 ou murmure vocal) qui correspond aux vibrations des cordes vocales et se concrétise par une concentration d'énergie dans les fréquences très graves (Figure 5). Les voyelles sont caractérisées par des formants (partie stable) ayant une certaine durée (souvent supérieure à $150 \mathrm{~ms}$ ) (Figure 5).

On appelle indice acoustique un paramètre physique du signal de parole dont les variations font changer l'identité phonétique des sons perçus. Les indices les plus significatifs sont ceux dont les variations changent le plus efficacement l'identité des sons perçus, mais qui varient le moins pour un phonème donné, en fonction du contexte et du locuteur. Des études perceptives ont montré que les unités phonétiques n'étaient pas en correspondance biunivoque avec des segments du signal de parole. Ainsi, même une bouffée de bruit centrée sur $1400 \mathrm{~Hz}$ peut être perçue comme un [p] devant un [i] et comme un [k] devant un [a] (Liberman et al. 1952). De même, une même transition de formant peut contribuer à la perception d'un [d] ou d'un [n] suivant le contexte acoustique. C'est pourquoi, l'identification des unités phonétiques peut nécessiter la prise en compte de plusieurs indices acoustiques répartis dans un contexte temporel plus ou moins large.

L'étude des erreurs d'identification des sons de parole dans le bruit montre que certaines distinctions phonétiques sont plus résistantes que d'autres. Pour les consonnes en contexte vocalique, les distinctions de nasalité et de voisement sont plus résistantes (Miller \& Nicely 1955). Viennent ensuite les distinctions entre occlusives et fricatives. Les distinctions de lieu d'articulation ([p] / [t] par exemple) sont celles qui sont le plus affectées par le bruit. "Cette sensibilité différentielle des sons de parole au bruit peut être considérée comme une validation perceptive de la notion de trait distinctif, les confusions se faisant entre les consonnes ayant le plus de traits en commun » (Calliope 1989). 

début du bruit d'explosion et l'établissement du voisement (Voice Onset Time - VOT - ou Délai d'Établissement du Voisement) était un indice essentiel pour la classification perceptive des occlusives voisées / non-voisées (Liberman et al. 1958). Ce délai de voisement représente l'intervalle entre le relâchement de l'air bloqué par le conduit vocal avant l'ouverture de la bouche et le déclenchement des vibrations des cordes vocales. Les VOT peuvent être positifs (voisement commençant après le relâchement de l'air bloqué dans le tractus vocal, [ph] anglais) ou négatifs (voisement commençant avant le relâchement de l'air, [b] français).

Figure 6. Le continuum du VOT en anglais et en français (Hesling 2002)

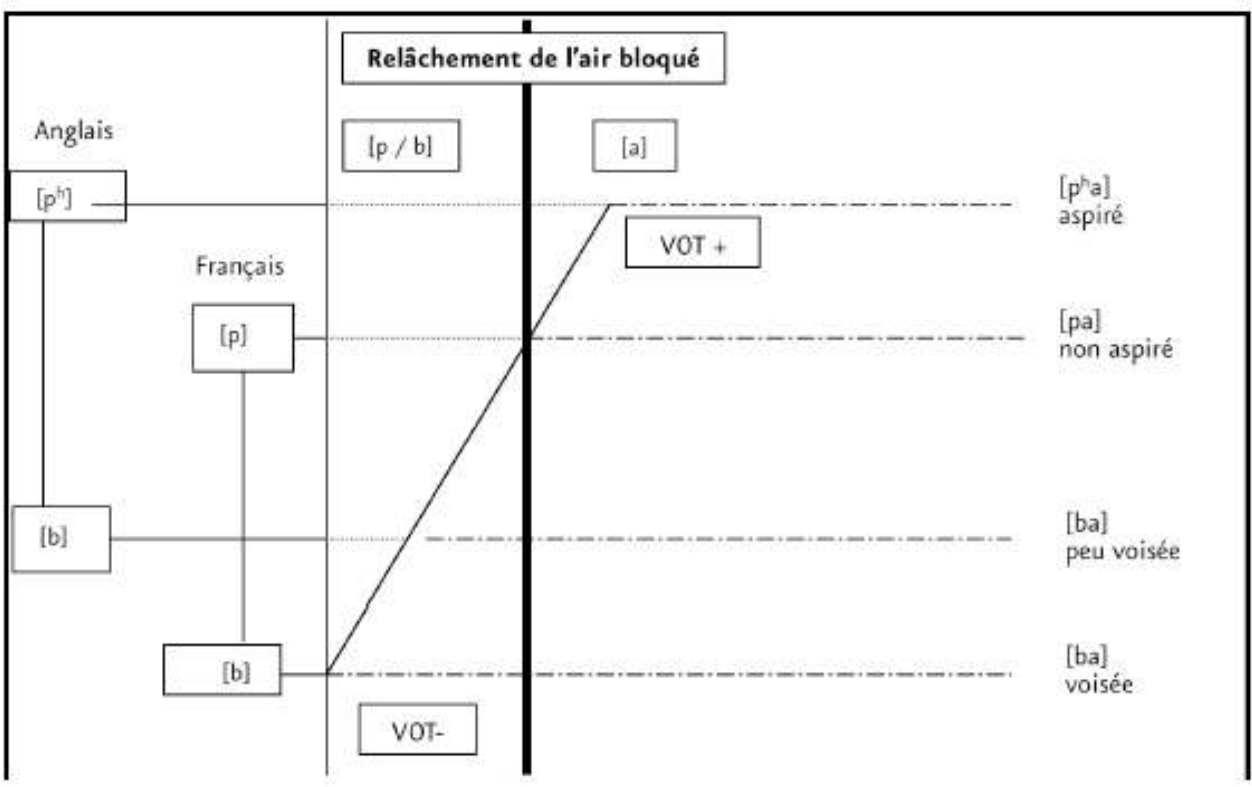

Les durées du VOT diffèrent en fonction des langues (Figure 6), par exemple, le [p] français a un VOT situé aux alentours de $20 \mathrm{~ms}$, le [ph] anglais présente un VOT d'une durée de $60 \mathrm{~ms}$. Le [b] français se situe aux alentours de $-100 \mathrm{~ms}$, le $\{\mathrm{b}$ ] anglais, vers $20 \mathrm{~ms}$. Ces données théoriques prennent toute leur importance en ce qui concerne les difficultés perceptives et productives pour les apprenants de langues étrangères.

\subsection{Neuropsychologie}

\subsubsection{Description anatomique du cerveau}

Un cerveau humain, représentant un quarantième du poids de l'individu, est formé de deux hémisphères accolés par leur face interne et séparés par une longue et profonde scissure inter-hémisphérique. Au fond de cette scissure se trouve une masse de fibres blanches appelée le corps calleux unissant entre eux les deux hémisphères. Ce corps calleux est constitué de fibres unissant les régions corticales homologues de chacun des hémisphères. Il existe d'autres voies de communication, moins importantes, entre les deux hémisphères (commissure de l'hippocampe et commissure antérieure). Le grand axe du cerveau s'étend du pôle frontal, en avant, au pôle occipital, en arrière. À la surface, de nombreuses fissures constituent un complexe réseau de circonvolutions. Les plus nettement marquées sont la scissure de Sylvius et la scissure de Rolando, qui permettent 
de différencier quatre lobes pour chaque hémisphère : lobe frontal, lobe pariétal, lobe temporal et lobe occipital.

\subsubsection{Asymétries morphologiques} oreille par l'intermédiaire d'écouteurs, provoquant ainsi une compétition entre chaque oreille et de ce fait entre chaque hémisphère cérébral, chaque oreille étant reliée préférentiellement à l'hémisphère cérébral opposé (Kimura 1967).

\section{hémisphère cérébral par injection d'amytal de sodium dans la carotide ipsilatérale, afin de le désactiver, ce qui permet d'observer le fonctionnement de l'autre hémisphère en isolation.}


ont été supprimées, permet d'étudier chaque hémisphère fonctionnant en isolation (Sperry 1968 ; Gazzaniga et al. 1975).

L'observation de sujets hémisphérectomisés (ablation chirurgicale d'un hémisphère) permet d'étudier le fonctionnement d'un hémisphère isolé (Smith 1977). Tous ces moyens d'observations chez les sujets sains et pathologiques apportent des informations concordantes sur les spécificités fonctionnelles de chaque hémisphère cérébral.

\subsubsection{Asymétries fonctionnelles}

Le tableau 1 résume les compétences spécifiques de chaque hémisphère cérébral en isolation : la colonne de gauche présente un inventaire d'activités mentales gérées par le cerveau total, la colonne du milieu indique les compétences et les incompétences de l'hémisphère gauche en isolation, c'est-à-dire fonctionnant sans connexions avec son homologue droit. La colonne de droite indique de même les compétences et les incompétences de l'hémisphère droit en isolation. Bien entendu, ce tableau est une compilation théorique du fonctionnement des deux hémisphères obtenue par les différentes techniques d'investigation sur des sujets sains et cérébro-lésés; en effet les deux hémisphères cérébraux ne fonctionnent pas de manière isolée mais en collaboration.

Tableau 1. Parole et cerveau : rôles respectifs des hémisphères cérébraux (Hesling 2000, d'après Déglin 1976)

\begin{tabular}{|l|l|l|}
\hline ACTIVITES de PAROLE & HG isolé & HD isolé \\
\hline PERCEPTION & & \\
\hline Répétition & Facile & Très réduite \\
\hline Identification des voix & Très difficile & Aisée \\
\hline Intonation & Non identifiée & Bien identifiée \\
\hline & & \\
PRODUCTION & & \\
\hline Lexique & Riche & Pauvre \\
\hline Syntaxe & Correcte & Très réduite \\
\hline Désir de communication & Volontaire & Très réduit \\
\hline Intonation & Plate & Excellente \\
\hline
\end{tabular}

En ce qui concerne la perception de la parole, la répétition est facile pour l'hémisphère gauche isolé (la répétition correcte étant la preuve d'une perception correcte), la reconnaissance des voix et le décodage de l'intonation sont de la compétence de l'hémisphère droit. Au niveau de la production de parole, le lexique, la syntaxe, sont des fonctions de l'hémisphère gauche, tandis que l'intonation est gérée par l'hémisphère droit. Sur le plan général du fonctionnement cérébral, il semble que l'on puisse qualifier les compétences de l'hémisphère gauche comme analytiques et celles de l'hémisphère droit comme globales ou synthétiques (Cambier \& Vertischel 1998). Sur le plan du développement cérébral, l'hémisphère droit du jeune enfant est en avance de développement fonctionnel sur son hémisphère gauche. Lors de l'acquisition de la langue maternelle, les deux hémisphères cérébraux sont sollicités, mais l'hémisphère droit reçoit manifestement une sollicitation plus importante que l'hémisphère gauche :

The right brain hemisphere seems to be more greatly affected by early experience than the left brain [...]. In an infant, the right brain hemisphere and the limbic 
system are initially dominant in regards to vocal communication. The right brain mental system does not rely on linguistic forms of analysis. (Joseph 1992) propre à l'espèce humaine. La parole est le résultat des divers processus neurologiques mettant en œuvre les compétences analytiques de l'hémisphère gauche ainsi que les compétences synthétiques de l'hémisphère droit (Figure 7). Les divers dispositifs physiologiques déclenchés par l'activité des deux hémisphères cérébraux sont à l'origine de phénomènes acoustiques spécifiques : l'hémisphère droit gère principalement la mise en vibrations des cordes vocales, vibrations donnant naissance à la fréquence fondamentale ( $\mathrm{F} 0)$, l'hémisphère gauche gère principalement la configuration de la cavité pharyngale (F1) et de la cavité buccale (F2).

Figure 7. Neurologie, acoustique et linguistique au service de la parole (Hesling 2002)

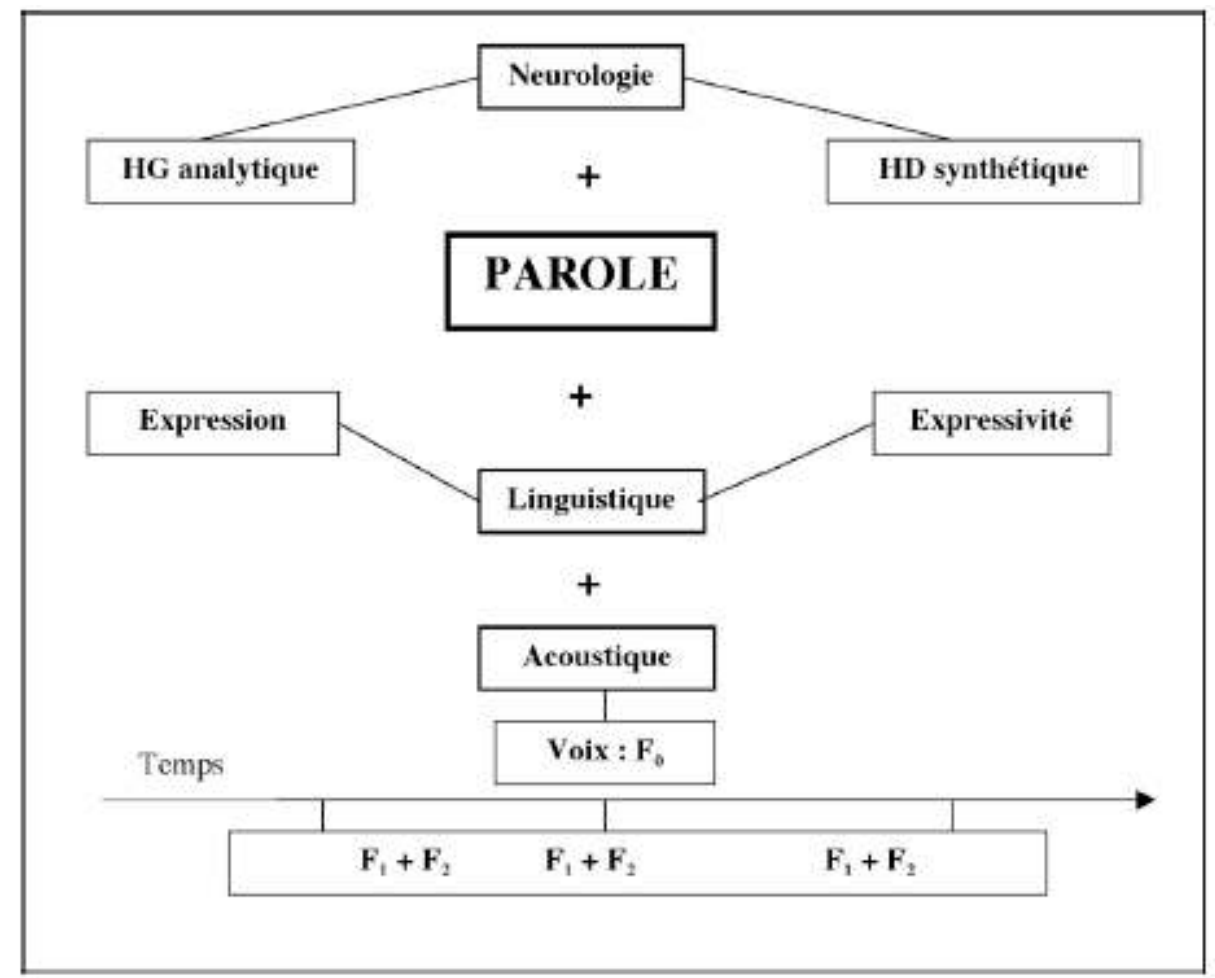


37 La fréquence fondamentale, support dynamique de la parole articulée, présente une certaine durée car la parole est bel et bien un continuum sonore et non pas une suite d'événements indépendants se déroulant sur l'axe des temps comme Matthei \& Ropper (1983) l'expliquent : « a beads-on-a-string model for speech perception could not work [...] and a beads-on-a-string model for speech production cannot work either ».

D'un point de vue linguistique, la signification par la parole résulte de la somme de la dimension expression et de la dimension expressivité.

Pour résumer, les éléments constitutifs de la parole, gérés tant d'un point de vue perceptif que productif par l'hémisphère gauche se synthétisent en parole grâce au pouvoir cohésif de l'hémisphère droit. La dimension émotionnelle, l'affectivité, portées par les variations de la fréquence fondamentale gérées par l'hémisphère droit, sont des éléments déclencheurs de parole.

\section{Recherche appliquée}

\subsection{Conditionnement neurophysiologique perceptif}

L'enfant est en contact avec sa langue maternelle dès sa naissance, et même in utero, puisque le fœtus, par l'intermédiaire du liquide amniotique, présente des réactions auditives à la voix maternelle et aux fréquences basses (Querleau \& Renard 1981). Dès sa naissance, il distingue la voix maternelle et localise tout phénomène sonore. À deux semaines, il différencie la voix humaine des autres émissions sonores. A partir de trois mois, il devient sensible aux inflexions de la voix humaine - c'est-à-dire à la prosodie qui correspondent à un contenu sémantique interprétable : il pleure lorsque la voix est rude et que ses intonations traduisent le reproche ou la colère, il rit lorsque les intonations sont tendres et bémolisées de complicité. À quatre mois, il est capable de différencier les productions vocales masculines et féminines (Petit 1992). Il est aussi capable de distinguer un nombre croissant de sons utilisés dans toutes les langues humaines (Werker \& Tees 1983). L'enfant se familiarise avec les intonations et les rythmes particuliers à sa langue. Il commence à communiquer sur cette base prosodique : c'est la période du babillage. Ce qui revient à dire que l'étape première dans l'apprentissage d'une langue, consiste en l'acquisition des rythmes et des intonations (la musique de la langue), ce que recouvre essentiellement le terme expressivité de la parole. Mais l'apprentissage de la langue maternelle n'exploite qu'une partie des potentialités inscrites dans le code génétique de l'enfant. Certaines oppositions sonores non pertinentes dans son milieu linguistique, vont devenir de moins en moins sensibles à son « oreille » (Figure 8). En fait, l'absence de stimuli induit une sclérose des synapses qui leur correspondent, c'est-à-dire qu'une « stabilisation sélective des synapses » s'effectue (Petit 1992). Le cerveau peut être comparé à un crible qui ne laisse passer que les éléments informationnels. Les sons non pertinents à la langue maternelle sont donc laissés de côté : «les sons de la langue étrangère reçoivent une interprétation phonologiquement inexacte, puisqu'on les fait passer par le 'crible phonologique' de sa propre langue » (Troubetzkoy 1949). Le cerveau opère par choix, par élimination: lors du processus d'identification des phonèmes, il filtre certains éléments physiques de l'ensemble fréquentiel et en retient certains autres. «Cette discontinuité dans l'écoute des sons du langage et le caractère particulier du choix des bandes de fréquences discontinues, dans chaque langue, forment le système d'écoute des sons du langage » (Gubérina 1991). 
Figure 8. Conditionnement neurophysiologique perceptif à la langue maternelle (Hesling 2000)

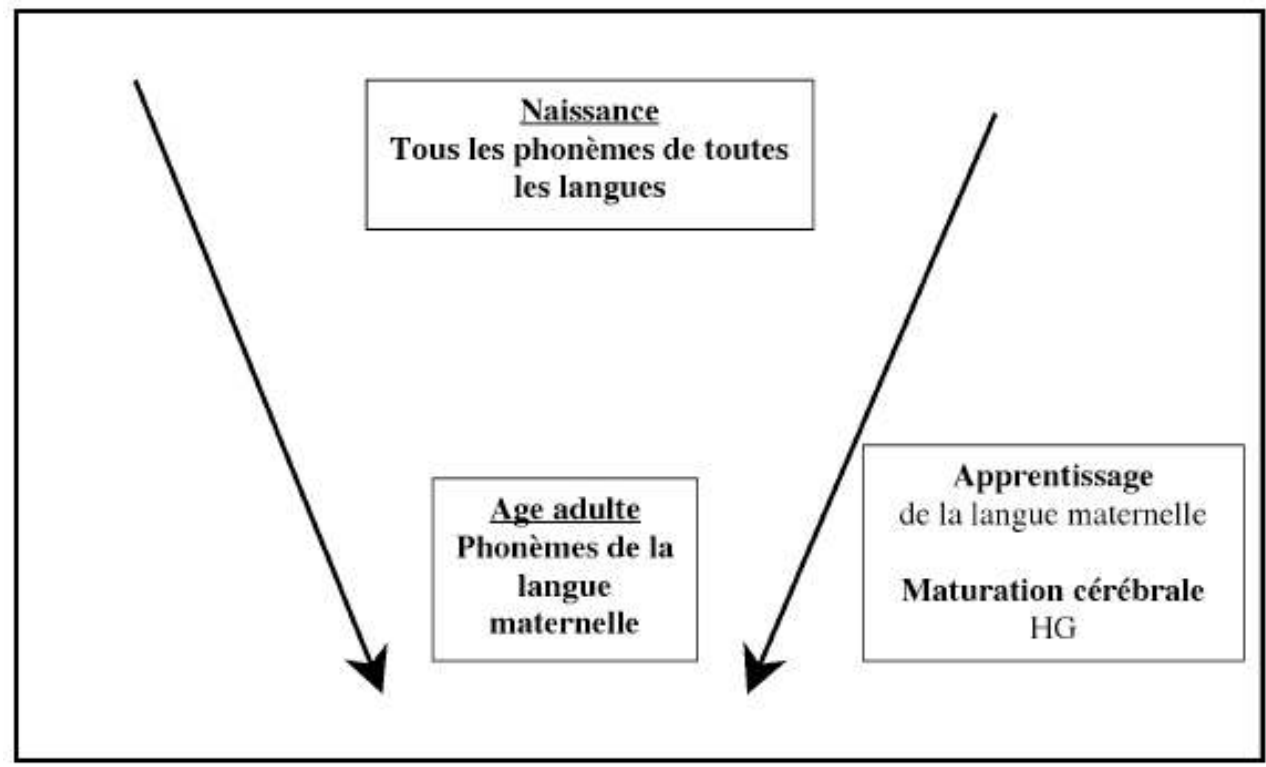

Le cerveau apte à décoder tous les phonèmes à la naissance, présente une grande flexibilité jusqu'à l'âge de 6-7 ans car il est en cours de croissance et de maturation. En revanche, après l'âge de sept ans, commence la phase de maturation des zones secondaires et tertiaires (et donc des centres de la parole de la langue maternelle). Selon Luria (1966) la myélinisation des zones secondaires et tertiaires est définitivement achevée à l'âge de 12 ans, ce qui explique d'un point de vue neurophysiologique et neuropsychologique qu'à partir de 12 ans cette maturation amène le cerveau à se comporter auditivement en fonction de sa langue première. Penfield (1963, in Gubérina 1991) souligne que « à 8 ans l'enfant commence à saisir les ensembles, à 9 ans il les fixe. Il passe pour devenir plus analytique dans son apprentissage de la langue ». Plus l'enfant mûrit dans son intelligence et dans sa psychomotricité, plus les facilités de l'acquisition d'une langue étrangère diminuent car plus il devient réfractaire aux sons de la langue étrangère: "sa langue maternelle affaiblit et restreint en partie sa sensibilité auditive [...]. L'acquisition d'une deuxième langue va inexorablement en sens inverse du progrès neuropsychologique de l'individu» (Gubérina 1990). Les habitudes linguistiques de la langue maternelle (qu'elles soient acoustiques ou articulatoires) sont donc ancrées vers l'âge de 10-12 ans, figeant ainsi la perception et de ce fait la production. Cependant, une bonne perception est la condition sine qua non à une bonne production : " la surdité est mère de la mutité » (Alliaume 1998). Pour prononcer correctement, c'est-à-dire pour parler de la manière la plus authentique possible, il est nécessaire d'avoir "entendu » (perçu) le modèle le plus correctement possible. Il est en effet vain de faire répéter inlassablement un mot mal prononcé ou d'expliquer comment positionner la langue contre les dents. Le processus de phonation met en jeu beaucoup d'autres « organes » qui sont incontrôlables, comme par exemple le larynx, les cordes vocales, quelques centaines de muscles... Le seul remède est d'amener l'apprenant à mieux percevoir le son fautif. D'un point de vue acoustique, la production des sons du langage est comprise dans une bande de fréquences allant de 20 à $20000 \mathrm{~Hz}$. D'un point de vue perceptif, l'analyse des sons du langage par un système de filtres octaves met en évidence le fait que chaque son contient potentiellement plusieurs phonèmes. Ainsi la voyelle française [i] est perçue 
comme $[\mathrm{u}]$ quand elle est transmise entre 150 et $300 \mathrm{~Hz}$, comme [o] entre 400 et $800 \mathrm{~Hz}$, comme [ə] entre 600 et $1200 \mathrm{~Hz}$, comme [a] entre 800 et $1600 \mathrm{~Hz}$, comme [e] entre 1200 et $2400 \mathrm{~Hz}$ puis comme [i] entre 3200 et $6400 \mathrm{~Hz}$. Ce phénomène s'applique à tous les sons de toutes les langues.

Gubérina et ses collaborateurs (1963) parviennent donc à la conclusion que chaque phonème contient potentiellement tous les autres phonèmes. La compréhension au sein d'une même langue ne s'en trouve nullement affectée mais les problèmes surviennent lors de l'apprentissage d'une langue étrangère. Une bonne audition permet une bonne perception des phonèmes de la langue 1 (langue maternelle) mais n'est plus du tout déterminante quant à la juste perception des phonèmes de la langue 2. En fait, elle commande même des fautes de substitutions dans la perception des phonèmes étrangers en créant de nouveaux systèmes de perception de ces phonèmes. Un son de la langue 1 présente une partie commune et une partie non commune avec un son de la langue 2 : le cerveau, conditionné par la langue 1 , ne retient que la partie commune à ces deux sons laissant de côté la partie non commune (partie néanmoins spécifique du son étranger). Cela amène donc l'interlocuteur de langue 1 à ne pas percevoir la spécificité du son de la langue 2 et donc à ne pas la reproduire (Figure 9).

Figure 9. Spectres du [i] anglais et du [i] français (Hesling 2000)

\section{Fréquence $(\mathbf{H z})$}

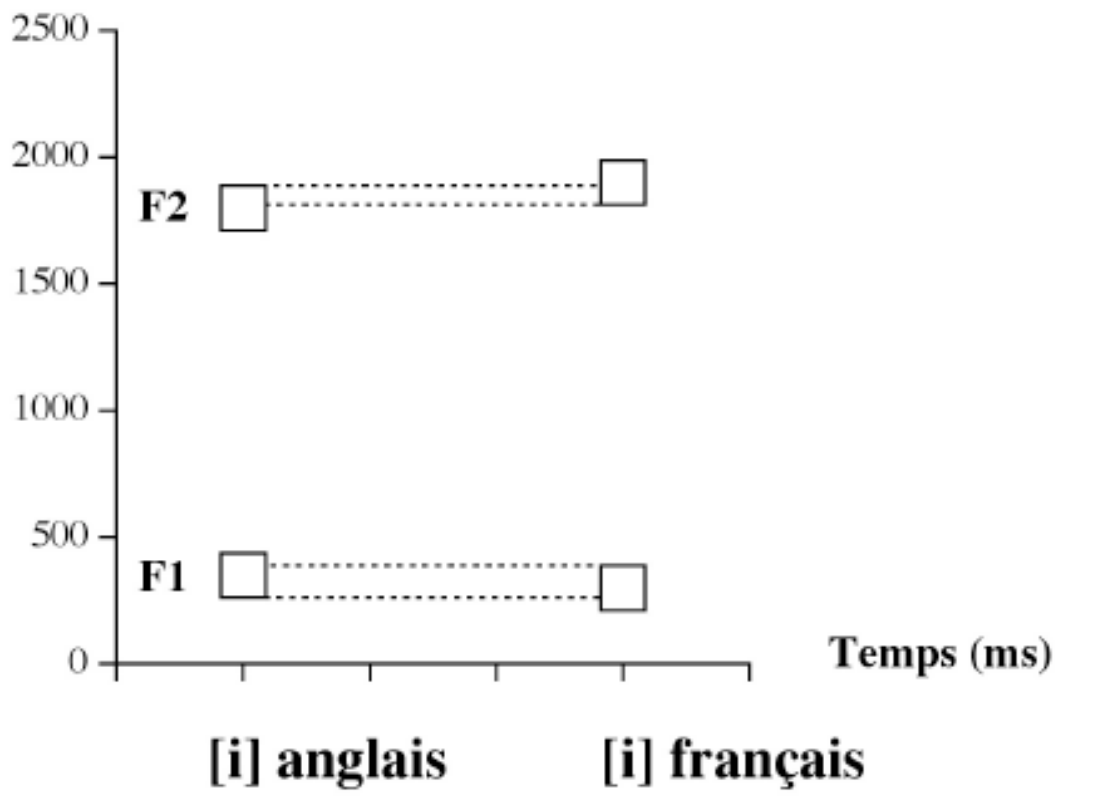

C'est la raison pour laquelle un anglophone perçoit le [y] français comme un [u] car il ne capte que les fréquences graves de ce [y] laissant de côté les fréquences aiguës (néanmoins spécifiques).

Tous les peuples ont la même acuité auditive pour les graves et pour les aiguës, mais n'ayant pas certains phonèmes, les différents peuples qui possèdent des systèmes linguistiques différents entendent différemment les mêmes sons d'une langue étrangère, pour des raisons psycholinguistiques (et non pas auditives) (Gubérina 1991) 
de parole. Dans un premier temps, ces ordres permettent une activité musculaire gérant la respiration et, linguistiquement parlant, les groupes de souffle, mais ces groupes de souffle sont calqués sur ceux de la langue maternelle. Puis, de manière plus spécifique, l'hémisphère cérébral droit va être majoritairement déclencheur de la mise en vibrations des cordes vocales, c'est-à-dire de la fréquence fondamentale et de ses variations. Là encore, les mises en vibrations sont calquées sur les caractéristiques de la langue maternelle. L'hémisphère gauche, majoritairement responsable de la configuration des cavités pharyngale et buccale ainsi que des organes phonatoires visibles responsables des mouvements articulatoires (lèvres, dents, langue), dirige ces organes selon les caractéristiques de la langue maternelle (Figure 10).

Figure 10. Conditionnement neurophysiologique productif à la langue maternelle (Hesling 2002)

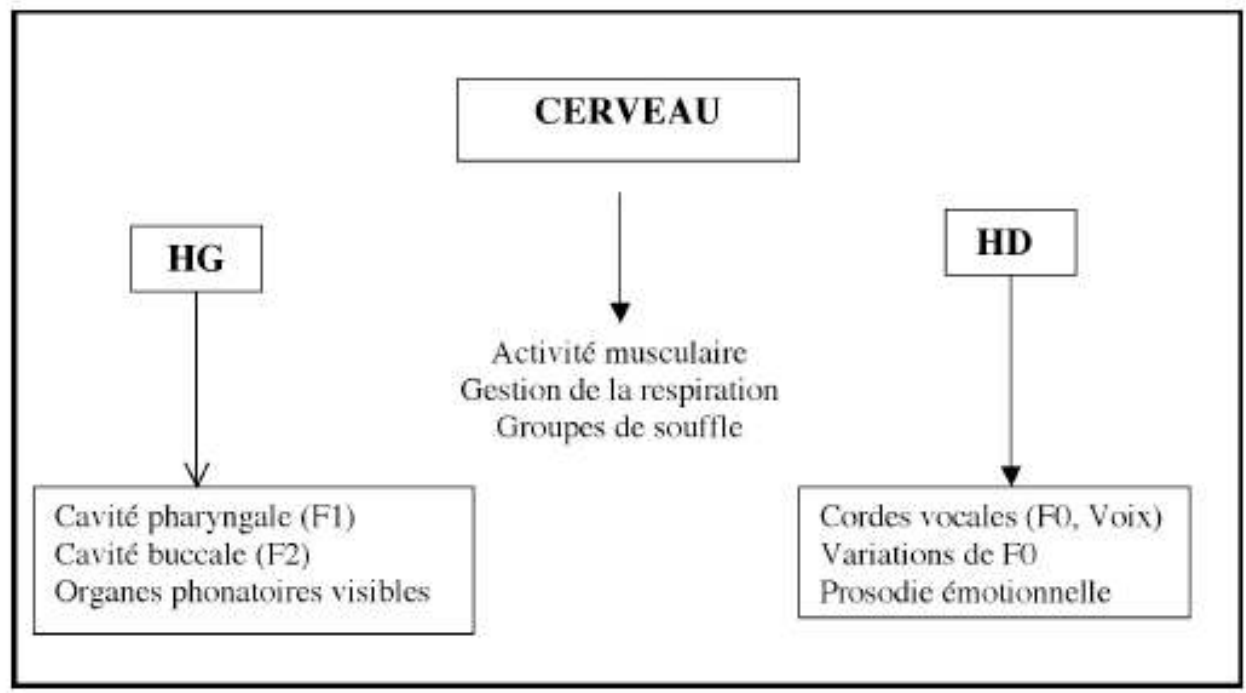

Un schéma corporel vocal particulier à chaque langue se constitue chez tout individu par mémorisation des données sensorielles non conscientes en relation avec les activités de parole en langue maternelle. En ce qui concerne la dimension prosodique, il convient, en langue anglaise, de différencier deux types de prosodie : la prosodie émotionnelle et la prosodie linguistique (Hesling \& Alliaume 2000). Chaque mot de deux syllabes ou plus est accentué, c'est le 
"stress " linguistique. Ce "stress » est une composante du mot au même titre que les phonèmes, qu'il soit indicateur de différences grammaticales ou non. Le "pattern" linguistique global est déterminé par la succession des «stress » des mots (lexicaux ou grammaticaux) qui composent l'énoncé et la gestion temporelle des groupes de souffle. Cette composante prosodique linguistique dépend de la langue et demeure indépendante de l'énonciateur. Les recherches récentes utilisant l'Imagerie par Résonance Magnétique fonctionnelle (Barrett et al. 1999; Riecker et al. 2002) montrent la supériorité de l'hémisphère gauche dans le traitement de la prosodie linguistique.

L'hémisphère droit surimpose au "pattern » linguistique géré par l'hémisphère gauche, la composante émotionnelle qui se traduit d'un point de vue acoustique par des variations de la fréquence fondamentale et de variations de l'intensité. Ces variations sont la marque personnelle de l'énonciateur sur son discours. Les émotions sont universelles mais l'impact des émotions sur le discours se traduit de manière spécifique en fonction des caractéristiques des langues maternelles. Cette composante prosodique émotionnelle dépend de l'état émotionnel de l'énonciateur et est principalement traitée par l'hémisphère droit (Barrett et al. 1999 ; Riecker et al. 2002).

\subsection{Conditionnements neurophysiologiques et pratiques scolaires}

Ces conditionnements neurophysiologiques perceptif et productif résultant de l'apprentissage de la langue maternelle sont de ce fait inévitables et expliquent partiellement les difficultés à maîtriser la parole en langue étrangère. Les pratiques pédagogiques offertes aux apprenants ne font que renforcer ces conditionnements (Figure 11). L'apprentissage de la langue maternelle permet un accès direct aux modalités verbales et non verbales, c'est-à-dire aux dimensions expression et expressivité de la parole ; les deux hémisphères cérébraux sont de ce fait sollicités. L'apprentissage scolaire de la langue étrangère entraîne un accès indirect aux dimensions expression et expressivité de la langue étrangère, puisque ces pratiques pédagogiques sont essentiellement orientées vers une sollicitation de l'hémisphère gauche. Cette orientation majoritairement hémisphérique gauche de l'enseignement des langues dans notre système scolaire semblerait pouvoir garantir au moins un accès satisfaisant à la dimension expression, mais le langage ainsi produit est réduit à sa composante purement intellectuelle puisque l'hémisphère droit, déclencheur et décodeur des manifestations émotionnelles, n'est que peu, voire pas, sollicité.

51 Figure 11. Sollicitation cérébrale, apprentissage de la langue maternelle, apprentissage scolaire d'une langue étrangère (Hesling 2000) 

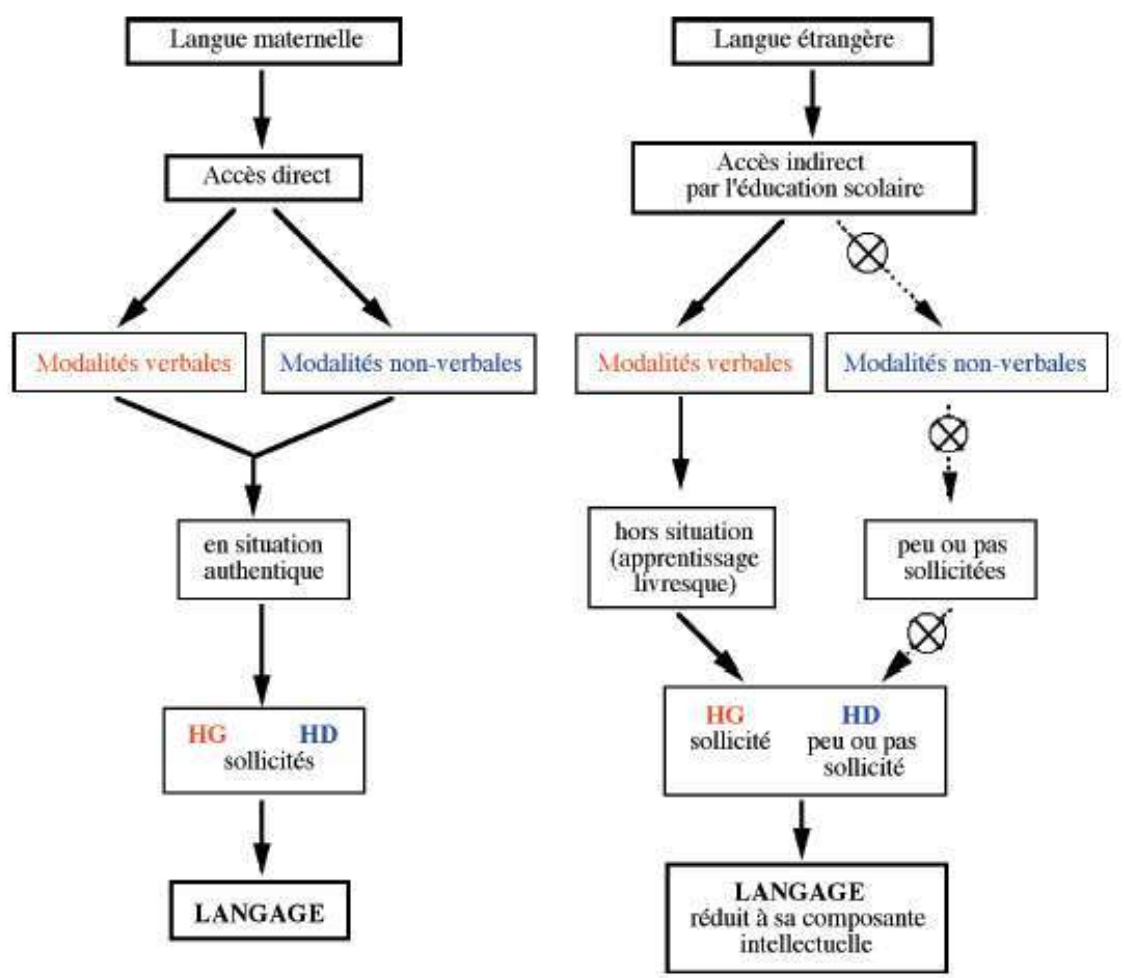

En résumé, les apprenants en langue de plus de dix ans sont confrontés à divers problèmes :

1. L'acquisition progressive de la maturité cérébrale entraîne un renversement de la situation dominante de l'hémisphère droit sur l'hémisphère gauche, l'hémisphère gauche prenant en quelque sorte le pouvoir et exerçant sa contrainte sur l'hémisphère droit, limitant ses compétences prosodiques perceptives et productives.

2. La maîtrise progressive de la langue maternelle entraîne un véritable conditionnement neurophysiologique, les organes de parole et, surtout, les zones cérébrales dévolues au langage perdant leur plasticité naturelle aux dépens des contraintes de fonctionnement de la parole maternelle.

3. Les pratiques pédagogiques proposées exercent essentiellement une sollicitation de l'hémisphère gauche.

Lorsqu'un apprenant écoute un message en langue étrangère, il se concentre essentiellement, pour ne pas dire uniquement, sur l'accès au sens et non sur la dimension prosodique de l'énoncé. Et lorsqu'un enseignant demande à un élève francophone, placé dans ces conditions, de répéter ce qui a été dit par l'interlocuteur anglophone, celui-ci tente de restituer le contenu sémantique, au détriment du contenu prosodique pourtant capital pour la communication. La réaction première de l'apprenant qui entend un message en langue étrangère est de tenter d'en décoder le sens. Le sens est, pense-t-il, transmis par les mots qui composent l'énoncé, ce qui dicte sa stratégie: il tente de déchiffrer les « mots » lexicaux et grammaticaux du message.

C'est clairement le type même de la stratégie hémisphère gauche, stratégie qui le prive des apports souvent considérables du contenu non verbal du message (affectivité, intonation, timbre) et qui, par conséquent, contribue à démobiliser son hémisphère droit. Comme l'affirme Cambier (1998),

Dans la mesure où l'apprentissage est perçu comme une tâche intellectuelle théorique affrontée hors du contexte, il détermine une activation élective de 
l'hémisphère gauche au détriment de l'hémisphère droit dont la participation est pourtant indispensable au fonctionnement de la communication.

\subsection{Propositions et perspectives}

55 Le concept de déséquilibre cérébral, naturel ou provoqué institutionnellement (par les pratiques pédagogiques inadaptées), s'applique à toutes les étapes du développement linguistique de l'individu apprenant de langues. Il peut être positivement utilisé par les pédagogues à tous les niveaux de développement cérébral : utilisation naturelle chez les tout-petits du développement précoce de l'hémisphère droit, stimulation artificielle de l'hémisphère droit, sous-utilisé chez les adultes.

Nos étudiants sont confrontés aux trois handicaps préalablement cités quant à l'apprentissage de la langue anglaise, à savoir: la maturation cérébrale (l'hémisphère gauche exerçant sa contrainte sur l'hémisphère droit); le conditionnement à la langue maternelle (surdité phonologique perceptive et donc productive aux phonèmes étrangers) ; les pratiques pédagogiques inadaptées (ces dernières ayant essentiellement exercé une sollicitation de l'hémisphère gauche au détriment de l'hémisphère droit).

Même si nos étudiants souffrent de ce déséquilibre cérébral inné et acquis, nous est-il encore possible de leur proposer des stratégies d'éducation, voire de rééducation? L'apprentissage de la langue maternelle constitue un atout psychologique dans le sens où chaque apprenant de langue étrangère a accompli, grâce à l'acquisition de sa langue maternelle, le passage essentiel du stade de la perception immédiate à celui de la conceptualisation (Vigotsky 1962 ; Luria 1966 ; Rosenfield 1989). Ainsi, contrairement au jeune enfant qui s'approprie progressivement la langue de son environnement, les apprenants adultes sont intellectuellement prêts à aborder les mécanismes nouveaux de l'anglais. L'atout constitué par la maturation intellectuelle, qui permet d'accéder à la compréhension et à la conceptualisation de mécanismes grammaticaux, voit sa portée singulièrement limitée par le conditionnement perceptif inhérent à la langue maternelle.

Le défi à relever est donc de déconditionner neurophysiologiquement nos apprenants. Pour ce faire, il apparaît primordial de solliciter activement et prioritairement leur hémisphère droit afin que les séquences prosodiques mises en place deviennent des lieux d'accueil privilégiés des séquences linguistiques. Mais comment ? Les quatre savoir-faire connus de tous les didacticiens de langues doivent impérativement être acquis dans cet ordre, l'oralité étant le point de départ de tout apprentissage communicationnel: compréhension orale; production orale; compréhension écrite; production écrite. Toutefois, à la lumière des connaissances en neuroacoustique, il semblerait judicieux de commencer l'acquisition de ces quatre savoir-faire par un travail de perception orale, celui-ci entraînant la compréhension orale. Il s'agirait donc d'acquérir une nouvelle compétence, la perception orale, visant à amener les étudiants à s'approprier la dimension prosodique de l'énoncé, sans tenter, dans un premier temps, d'en décrypter le contenu sémantique. Puis, lorsque la dimension prosodique, support dynamique de la parole, serait en place, les étudiants pourraient ensuite se concentrer sur le sens.

Comment faciliter l'acquisition de cette nouvelle compétence (la musique de la parole étrangère)? Des pratiques pédagogiques essentiellement orientées dans un premier temps vers une activation de l'hémisphère droit peuvent s'avérer utiles. En fait, il s'agit de proposer une approche essentiellement globale pour ensuite se focaliser sur l'analytique. Mais les contraintes pédagogiques auxquelles tous les enseignants sont 
confrontés - maximum de compétences à faire acquérir dans un minimum de temps - ne permettent pas de consacrer le temps nécessaire en vue d'une acquisition solide de cette dimension prosodique. Qui plus est, tous les exercices, si originaux soient-ils, peuvent, à la rigueur, amener nos apprenants à appréhender de manière analytique (hémisphère gauche) la dimension prosodique mais ne peuvent en aucun cas les amener à intégrer cette dimension prosodique de manière holistique (hémisphère droit). En d'autres termes, la nouvelle compétence dite "perception orale" ne peut pas être acquise efficacement par l'intellect. En résumé, toutes les propositions pédagogiques, aussi innovantes soient-elles, ne sont en fait qu'un pis-aller au vu des processus de rééducation nécessaires; processus nécessitant un déconditionnement neurophysiologique perceptif (entrânnant ainsi un déconditionnement neurophysiologique productif) à la langue maternelle.

60 Alors, comment déconditionner nos apprenants ? Ce déconditionnement ne peut pas se faire de manière raisonnée, c'est-à-dire en utilisant les seules aptitudes de l'hémisphère cérébral gauche. En effet comment demander à un apprenant d'entendre ce que son cerveau conditionné ne peut percevoir? Comment demander ensuite à ce même apprenant de reproduire ce qu'il n'a pas pu percevoir? Les maintes tentatives de la phonétique articulatoire se sont avérées vaines car il est bien sûr impossible de demander, de manière raisonnée, au seul hémisphère gauche de l'apprenant d'agir sur les quelques milliers de muscles gérant la production de parole. En fait, il s'agit de s'adresser directement, en-dessous du seuil de conscience de HG, à HD. Les propositions pédagogiques s'avérant peu efficaces pour mener à bien notre objectif, il s'agit donc de reconsidérer le problème à sa source, c'est-à-dire de revenir aux fonctionnalités cérébrales.

De nombreuses études mettent en lumière le rôle de l'hémisphère cérébral droit dans le traitement des émotions quelle que soit leur nature - gestuelles, faciales, lexicales, prosodiques (Bear 1983 ; Borod et al. 1996; Cummings 1997; Ross et al. 1997). D'autres travaux en Imagerie par Résonance Magnétique fonctionnelle concluent à une prédominance de l'hémisphère cérébral droit dans la perception de la prosodie (Bradvik et al. 1991 ; Pell \& Baum 1997). Ces recherches fondamentales, bien que confortant notre propos, n'apportent pas de solutions à notre problème.

Comment s'adresser directement à l'HD des apprenants en court-circuitant l'emprise exercée par leur HG? La notion de discontinuité de perception des sons de parole a permis à Gubérina de mettre au point un appareil destiné dans un premier temps aux déficients auditifs (Gubérina 1973). Ce système (le Suvag) permet d'adresser à chaque malentendant le champ auditif qui lui est optimal en s'adaptant à ses restes auditifs. Autrement dit, le Suvag fonctionne en discontinuité (certaines fréquences étant conservées, d'autres étant supprimées), se faisant ainsi le reflet du processus de discontinuité de la perception auditive tel qu'il existe chez les entendants. Faisant l'analogie entre pathologie de l'audition et apprentissage des langues, Gubérina fait évoluer le Suvag en Suvag-Lingua : « la discontinuité dans l'écoute des sons du langage et le caractère particulier du choix des bandes de fréquences discontinues dans chaque langue, forment le système d'écoute des sons du langage » (Gubérina 1973).

63 À la suite des travaux de Gubérina, un autre chercheur établit une passerelle entre la recherche neurologique, la recherche acoustique et la recherche linguistique, dans le but de permettre une utilisation plus optimale du potentiel de chacun en matière 
d'apprentissage des langues, de rééducation des troubles de la parole ou de rééducation des troubles communicationnels.

Le principe fondamental du dispositif Intolang (Alliaume 1989) est de solliciter simultanément les deux hémisphères cérébraux en adressant à chacun, en fonction de ses spécificités fonctionnelles, les composantes des stimuli verbaux les plus optimales. Intolang permet d'adresser à $\mathrm{HD}$, spécialisé dans la gestion des modalités non-verbales, un message dépouillé de son contenu linguistique et réduit à sa dimension prosodique, c'est-à-dire de lui adresser la dimension affective du discours (manifestée par les modulations de F0). Simultanément HG, responsable des modalités verbales, reçoit le message dans la totalité de sa composante acoustique. Selon le principe de l'écoute dichotique (Kimura 1967), c'est-à-dire un message différent à chaque oreille, donc à chaque hémisphère cérébral, via les connexions croisées, Intolang adresse au cerveau total le contenu linguistique par l'intermédiaire de l'oreille droite (HG) et le contenu prosodique (extrait par filtrage acoustique) à l'oreille gauche (HD), de manière dissociée. Dans ces conditions, il semble que chaque hémisphère reçoive le message dans la modalité pour laquelle il est spécialisé.

Le problème de la communication directe avec l'HD d'une personne en dehors de la conscience de HG semble résolu par le système proposé. La domination exercée normalement par HG sur HD est ainsi court-circuitée puisque HG n'a aucune conscience du message adressé à HD. Il s'agit donc d'une coopération inconsciente exercée entre les deux hémisphères. En procédant ainsi - en fournissant à HG une information explicite (un message en clair) et à HD une information implicite (la composante prosodique du même message en dessous du niveau de conscience)- l'emprise de HG sur HD se trouve limitée et nous permettons au cerveau total de prendre en compte toutes les caractéristiques acoustiques du message. Or l'implicite, qui se manifeste par les variations de rythme et d'intonation, est ce qui donne de la réalité à l'explicite. La fonction primordiale de ce dispositif est de permettre à l'utilisateur d'intégrer l'implicite, inaccessible par le raisonnement. De nombreuses études cliniques réalisées, tant dans le domaine de l'apprentissage de l'anglais chez des sujets adultes que dans le domaine de la rééducation des troubles de la parole - aphasie, autisme - se sont avérées probantes (Hesling 2000).

Mais que se passe-t-il au niveau cérébral chez les utilisateurs de ce dispositif ? Leurs progrès notoires sont-ils dus à une plus grande activité de leur hémisphère droit, sursollicité, ou leur hémisphère droit sur-sollicité entraîne-t-il une plus grande activation de leur hémisphère gauche ? Une étude en IRMf chez des sujets sains (Hesling \& Allard 2001) tente de répondre à ce questionnement en visant à déterminer les canevas d'activation cérébrale lors de la perception de fréquences acoustiques de parole spécifiquement sélectionnées, cette activation étant appréciée selon deux critères, taille en surface des zones d'activation corticale et répartition topographique de ces zones d'activation au niveau de la surface corticale. En effet l'IRMf permet, en raison des propriétés magnétiques de l'hémoglobine, l'enregistrement des variations hémodynamiques induites par l'activation neuronale. Les premiers résultats semblent prometteurs.

La prise de conscience, par les enseignants de langue, qu'aucune pédagogie ne peut se concevoir sans une connaissance minimale du fonctionnement cérébral des apprenants, paraît constituer la voie incontournable pour un enseignement efficace. 


\section{BIBLIOGRAPHIE}

Alliaume, J. 1989. Procédé de traitement de certaines composantes acoustiques de la parole et appareil de mise en œuvre. Brevet d'invention, N² 642 557. Institut National de la Propriété Industrielle.

Alliaume, J. \& I. Hesling. 1998. Le langage et les deux cerveaux. Fort de France : Éditions du CERALEC.

Barrett, A. M., G. P. Crucian, A. M. Raymer \& K. M. Heilman. 1999. « Spared comprehension of emotional prosody in a patient with global aphasia ». Neuropsychiatry \& Neuropsychology 12/2, 117-120.

Bear, D. M. 1983. « La spécialisation hémisphérique et les fonctions émotionnelles chez l'homme ». Revue de neurologie 139, 23-33.

Borod, J. C., K. D. Rorie, C. S. Haywood, F. Andelman, L. K. Obler, J. Welkowitz, R. L. Bloom \& J. R. Tweedy. 1996. « Hemispheric specialization for discourse reports of emotional experiences: relationships to demographic, neurological, and perceptual variables ». Neuropsychologia 34, 351-359.

Bradvick, B., C. Dravins, S. Holtas, I. Rosen, E. Ryding \& D. Ingvar. 1991. « Disturbances of speech prosody following a right hemisphere infarcts ». Acta Neurol Scand, 114-126.

Calliope. 1989. La parole et son traitement automatique. Paris : Masson.

Cambier, J. \& P. Verstichel. 1998. Le cerveau réconcilié. Paris : Masson.

Cummings, J. L. 1997. « Neuropsychiatric manifestations of right hemisphere lesions ». Brain and Language 57/1, 22-37.

Deglin, V. 1976. « Voyage à travers le cerveau ». Le Courrier de l'UNESCO Paris, 4-20.

Ferrand, L. \& J. Ségui. 1996. « Produire et comprendre le langage parlé ». Sciences \& Vie hors série $195,146-156$

Gazzaniga, M. S, G. L. Risse, S. P. Springer, E. Clark \& D. H. Wilson. 1975. « Psychologic and neurologic consequences of partial and complete commissurotomy ». Neurology 25, 10-15.

Gil, R. 1996. Neuropsychologie. Paris : Masson.

Goleman, D. 1997. L'intelligence émotionnelle. Paris : Laffont.

Gubérina, P., Y. Gospodnetic, M. Pozojevic, I. Skaric \& B. Vuletic. 1963. « Correction de la prononciation des élèves qui apprennent le français ». Revue de phonétique appliquée 1, 81-93.

Gubérina, P. 1973. « Méthode verbo-tonale : méthode humaniste ». Bulletin des praticiens de la méthode verbo-tonale 1, 3-13.

Gubérina, P. 1984. « Bases théoriques de la méthode audio-visuelle structuro-globale. Une linguistique de la parole ». Aspects d'une politique de diffusion du français langue étrangère depuis 1945, 85-99. Saint-Cloud : Hatier.

Gubérina, P. 1990. «La surdité à bras le corps ». Tire ta langue, Chronique de Philippe Barthelet, France-Culture.

Gubérina, P. 1991. «Rôle de la perception auditive dans l'apprentissage précoce des langues ». In Garabedian, M, Enseignements / Apprentissages précoces des langues. Vanves : Hachette F.L.E. 
Habib, M. 1989. Bases neurologiques des comportements. Paris : Masson.

Hesling, I. 2000. « Le rôle de l'hémisphère cérébral droit dans la perception et la production de la parole. Études physico-acoustiques - Investigations neurolinguistiques. Propositions pour un rééquilibrage de l'activité cérébrale ». Thèse de doctorat, Université Toulouse 2.

Hesling, I.\& J. Alliaume. 2000. « Traitement cérébral des rythmes en parole anglaise ». Colloque International, Linguistique et langue anglaise / Linguistics and the English Language, Toulouse.

Hesling, I.\& M. Allard. 2001. « Activation cérébrale induite par l'audition des fréquences acoustiques spécifiques de la parole. Une étude en IRM $\mathrm{f}$ chez le sujet sain ». CCPPRB $n^{\circ}$ d'enregistrement 2001/42,17/08/2001.

Joly, A. \& D. O'Kelly. 1990. Grammaire systématique de l'anglais. Paris : Nathan.

Joseph, R. 1992. The right brain and the unconscious. New York : Plenum Press.

Kimura, D. 1967. « Functional asymmetry of the brain in dichotic listening ». Cortex 3/2, 163-178.

Liberman, A. M., P. C. Delattre \& F. S. Cooper. 1952. «The role of the selected stimulus variables in the perception of the unvoiced consonants ». Journal of the Acoustical Society of America 65, 497-516.

Liberman, A. M., P. C. Delattre \& F. S. Cooper. 1958. « Some cues for the distinction between voiced and voiceless stops ». Language and Speech 1, 153-167.

Luria, R.A. 1966. Higher Cortical Functions in Man. Londres : Tavistock.

Luria, R.A. 1970. « The functional organisation of the brain ». Scientific American 222, 66-78.

Matthei, E. \& T. Roeper. 1983. Understanding and Producing Speech. Bungay, Suffolk : Fontana Paperbacks.

Miller, G. A. \& P. Nicely. 1955. « An analysis of perception confusions among some English consonants ». Journal of the Acoustical Society of America 27, 338-352.

Pell, M. D. \& S. R. Baum. 1997. « The ability to perceive and comprehend intonation in linguistic and affective contexts by brain-damaged adults ». Brain and Language 57, 80-89.

Petit, J. 1992. Au secours, je suis monolingue et francophone! Presses Universitaires de Reims, Publications du Centre de Recherche en Linguistique et Psychologie cognitive, UFR des Lettres et Sciences Humaines de Reims.

Querleau, D. \& K. Renard. 1981. «Les perceptions auditives du foetus humain ». Médecine et hygiène 39, 2102-2110.

Riecker, A., D. Wildgruber, G. Dogil, W. Grodd \& H. Ackermann. 2002. « Hemispheric lateralization effects of rhythm implementation during syllable repetitions: An f MRI study ». Neuroimage 16/1, 169-176.

Renard, R. 1983. Mémento de phonétique à l'usage des professeurs de langues et des orthophonistes. Bruxelles : Centre International de Phonétique Appliquée/ Didier Hatier.

Robergé, C. 1992. «Mérites et démérites de la notion saussurienne de 'parole' ». Revue de Phonétique Appliquée 105, 339-353.

Rosenfield, I. 1989. L'invention de la mémoire. Paris : Eshel.

Ross, E. D., R. D. Thompson \& J. Yenkosky. 1997. « Lateralization of affective prosody in brain and the callosal integration of hemispheric language functions ». Brain and Language 56, 27-54.

Smith, A. 1977. « Language and the cerebral hemispheres ». Neurology 27, 627- 633. 
Sperry, R.W. 1968. « Hemispheric disconnection and unity in conscious awareness ». American

Phychologist 23, 723-733.

Troubetzkoy, N.S. 1949. Principes de phonologie, trad. fr. Paris : Éditions Klincksieck.

Vigotsky, L.S. 1962. Thought and Language. Cambridge, MA : The MIT Press.

Werker, J. F. \& R. Tees. 1993. « Developmental changes across childhood in the perception of nonnative speech sounds ». Canadian Journal of Psychology 37, 278-286.

\section{RÉSUMÉS}

À l'ère de la pluridisciplinarité, il semble fondamental que toute pratique pédagogique et/ou didactique tienne compte des apports des neurosciences - l'anglais de spécialité ne dérogeant pas à ce postulat. Tout apprenant de langue étrangère est conditionné linguistiquement (cérébralement) à sa langue maternelle et met en jeu ses deux hémisphères cérébraux, et ce quelle que soit sa préférence manuelle, lors de l'apprentissage d'une langue seconde. D'où l'intérêt de mieux connaître les rôles distinctifs, mais néanmoins complémentaires, dévolus à l'hémisphère droit et à l'hémisphère gauche en ce qui concerne le traitement cérébral de la parole. Bien que nombre d'études privilégient l'importance de l'hémisphère gauche dans l'acte de parole, le rôle capital de l'hémisphère droit ne peut être passé sous silence. Dans un premier temps, une synthèse des données obtenues en recherche fondamentale dans des domaines aussi variés que la linguistique, l'acoustique, la neurologie et la neuropsychologie, domaines divers s'avérant cependant très complémentaires, sera proposée. Puis, dans un deuxième temps, ces données théoriques et comportementales seront exploitées à des fins pédagogiques et didactiques, ceci afin de mieux cerner les problèmes des étudiants en anglais de spécialité et de tenter de mieux répondre à leurs attentes.

Any genuinely multidisciplinary approach in pedagogical and/or didactical practices needs to take into account the contributions of the neurosciences. Foreign language learners run into specific difficulties which can only be remedied if analysed from the point of view of neurolinguistics. Whatever his/her mastery of the foreign language and the use he has for it, each foreign language learner is linguistically - that is cerebrally - conditioned by his/her mother tongue and uses both his/her left and right cerebral hemispheres. The role of each cerebral hemisphere in the processing of speech must be clearly understood, since, even if they work in close collaboration, the left hemisphere and the right hemisphere do not process the same kind of speech information. Moreover, the crucial role of the right hemisphere in speech processing should not be ignored, in spite of the emphasis laid by most studies upon the role of the left hemisphere. The present paper will begin by a synthesis of fundamental research data in different but complementary fields such as neurology, acoustics, psycholinguistics and linguistics. Then, these theoretical data will be exploited from a pedagogical and didactical point of view and some solutions to the difficulties encountered by foreign language learners will be proposed.

\section{INDEX}

Mots-clés : acoustique, hémisphère cérébral droit, parole, prosodie

Keywords : acoustic, prosody, right cerebral hemisphere, speech 
AUTEUR

ISABELLE HESLING 